\title{
The relative roles of intellectual discourse and psychomotor performance in clinical medicine
}

\author{
Stephen A Margolis
}

No matter how well trained people are, few can sustain their best performance on their own. That's where coaching comes in. - Atul Gawande ${ }^{1}$

I have always been interested in the apparent dichotomy between the two arms of medical training and practice: the exploration of disease and illness through intellectual discourse versus the practical use of the clinician's psychomotor skills and techniques to physically interact with the patient. Perhaps there is no real surprise as medicine's two branches - the less invasive medical methods and the more invasive surgical methods - have been present since before the existence of written language. ${ }^{2}$ This duality persists in the designation of the primary medical degree in most countries as a combination of a Bachelor of Medicine with a Bachelor of Surgery.

Hence, medical graduates in recent times were required to be competent at two seemingly completely different tasks: the cerebral scientific understanding, interpretation and application of medical science to their patient's needs; and the development, refinement and application of the often complex physical movement, coordination, manipulation, dexterity, grace, strength and speed to perform surgical procedures. While a practitioner skilled at the former can manage a patient with cardiovascular risk factors, they may struggle with knot tying when performing even the most basic procedures. With the seemingly never-ending sub-specialisation within medicine, medical practitioners can excel in non-procedural niche areas that complement their innate abilities. Perhaps procedural areas are best for those who shine in psychomotor skills?

Where does this leave general practice? By definition, general practitioners (GPs) work across a broad range of clinical topic areas and are encouraged to develop and maintain physical procedural skills. Within this group of activities, management of wounds remains the core foundation skill and is equally applicable across the metro-rural divide. However, unlike non-procedural skills that can be learnt and developed within a 'reading - observing - simulated performance - discussion' environment, physical psychomotor skills additionally require real-life performance of the task within a coaching-feedback environment. Simulation has greatly decreased the burden placed on patients undergoing procedures by trainees but has not reduced this to zero.

With this in mind, the September issue of the Australian Journal of General Practice (AJGP) includes four articles on the management of minor wounds and burns that are commonly seen in general practice and well within the remit of GP management. Turner describes the assessment and management of minor wounds, ${ }^{3,4}$ Sinha additionally considers post-operative wounds, ${ }^{5}$ while Tran, Jacques and Holland discuss the assessment and management of minor paediatric burns. ${ }^{6}$ The intention is to provide an update on the core principles underpinning the evidence-based care of these common conditions, with the understanding that clinical competence of physical application of the techniques described will require hands-on teaching and mentoring. Rather than delve into less familiar and more esoteric areas, the goal here is to encourage GPs to actively engage in physical management of their patients. Apart from potentially decreasing the burden on their local emergency departments, incorporating broader-based wound care within their practices may enhance the GPs' connectedness with their patients in addition to the joy of having additional skills in their armamentarium.

GPs have traditionally provided clinical care for their patients across the gamut of clinical conditions using both verbal discourse and physical psychomotor skills. With the broader move to sub-specialisation in other medical disciplines, the focus has perhaps decreased on the latter. The articles in this edition of AJGP, together with thoughtful peer guidance and mentoring, will hopefully encourage GPs to grow the surgical/procedural side of their day-to-day clinical practice.

\section{Author}

Stephen A Margolis, OAM; Senior Medical Editor at Australian Journal of General Practice; Professor, School of Medicine, Griffith University, Qld; Senior Staff Specialist, Capricorn Coast Hospital, Qld; Medical Officer, Royal Flying Doctor Service.

\section{References}

1. Gawande A. Personal best: Top athletes and singers have coaches. Should you? The New Yorker. 3 October 2011. Available at www.newyorker.com/ magazine/2011/10/03/personal-best [Accessed 5 August 2019].

2. Aggarwal $A$. The evolving relationship between surgery and medicine. Virtual Mentor 2010;12(2):119-23. doi: 10.1001/ virtualmentor.2010.12.2.mhst1-1002.

3. Turner RC, Tran V. Acute lacerations: Assessment and non-surgical management. Aust J Gen Pract 2019;48(9):585-88

4. Turner RC. Surgical management of acute lacerations. Aust J Gen Pract 2019;48(9):600-03.

5. Sinha S. Management of post-surgical wounds in general practice. Aust J Gen Pract 2019;48(9):596-99.

6. Tran S, Jacques MA, Holland AJA. Assessment and management of minor burns in children. Aust J Gen Pract 2019;48(9):590-95. 